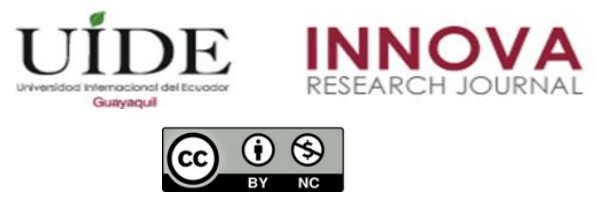

INNOVA Research Journal, ISSN 2477-9024

(Septiembre-Diciembre, 2019). Vol. 4, No.3 pp.131-144

DOI: https://doi.org/10.33890/innova.v4.n3.2019.977

URL: http://revistas.uide.edu.ec/index.php/innova/index

Correo: innova@uide.edu.ec

\title{
Referencias para calificar espacios comunes en ciudades de zonas metropolitanas del valle de Toluca, México
}

\section{References for classifying common spaces in cities in metropolitan areas of the valley of Toluca, México}

Laura Teresa Gómez-Vera

Verónica Zendejas-Santín

Georgina Alicia García Luna-Villagrán

Universidad Autónoma del Estado de México, México

Autor para correspondencia: lagov13@gmail.com; verozendejas3@gmail.com; ginamasa@hotmail.com

Fecha de recepción: 9 de abril del 2019 - Fecha de aceptación: 29 de julio del 2019

Resumen: El propósito del presente trabajo fue definir y entender los criterios que inducen a la conformación de indicadores que permitan comprender las variables relativas al derecho de cohabitar espacios comunes en condiciones sostenibles. Esto como una referencia para calificar ciudades de zonas metropolitanas con base en las teorías de habitabilidad, competitividad urbana y nuevo urbanismo. En términos metodológicos, se analizaron los ejes de permeabilidad, de variedad, de robustez, de legibilidad y de riqueza. De estos se desprendieron las dimensiones sociales, económicas y ambientales en las que se clasificaron las categorías de medición. Resultan ser señales del crecimiento inclusivo y de los principios de habitabilidad a favor de la competitividad urbana. Las aportaciones de este estudio se reflejan en la interpretación de dichas categorías que encamina al diseño de indicadores para delimitar el significado y el desempeño de proyectos y políticas institucionales. De esta manera se trabaja a favor de la toma de decisiones de los colectivos para mejorar la calidad de vida en las ciudades de zonas metropolitanas del valle de Toluca, México.

Palabras clave: gestión; espacios comunes; habitabilidad; sostenibilidad; indicadores

Abstract: The goal of the present work was to define and understand the criteria that lead to the formation of indicators in order to understand the variables regarding the right to cohabit common spaces in sustainable conditions. This is a reference of qualifying cities in metropolitan areas based on the theories of habitability, urban competitiveness and new urbanism. In methodological terms, the axes of permeability, variety, robustness, legibility and richness were analyzed. These show the social, economic and environmental dimensions in which the categories of measurement were classified. All are signs of inclusive growth and principles of habitability in favor of urban competitiveness. The results of this study are reflected in the interpretation of those categories that suggests the design of indicators to delimit the meaning and performance of institutional projects and policies. This work is in favor of the decision making of the groups to improve the quality of life in the cities of metropolitan areas of the valley of Mexico.

Key Words: management; common spaces; inhabitability; sustainability; indicators 


\section{Introducción}

La concentración de población que se ha manifestado en las ciudades medias del valle de Toluca, a raíz de la inestabilidad en el desempeño de las zonas rurales, ha provocado la expansión y la conurbación de la mancha urbana. En consecuencia se han formado nuevas zonas metropolitanas que muestran en su estructura ciertos obstáculos que impiden un buen desempeño social y productivo en los barrios o suburbios.

En las próximas décadas, el crecimiento demográfico será en gran parte urbano. Cabe mencionar que para el año 2030 la República Mexicana pasará de contar de 384 ciudades a 961, en las que se concentrará $83.2 \%$ de la población. Muy probablemente será aquella población en condiciones de pobreza la que predominará (ONU-Habitat, 2016). Esta tendencia, junto con la falta de parámetros que ayuden entender su configuración, obstaculiza el desarrollo que es deseado en beneficio de los colectivos sociales. 1

Estudiar el significado y la apreciación que origina nexos en los espacios comunes de centros urbanos tiene que ver con la línea de generación y aplicación del conocimiento [LGAC] «Sistemas y modelos de gestión para la innovación de proyectos de inversión y del ordenamiento del hábitat y su entorno».2 En lo particular, es de interés exponer las bases teóricas que son necesarias para diseñar indicadores que permitan evaluar las dimensiones [social, económica y ambiental] que están implícitas en el estudio de las condiciones que hacen que las áreas comunitarias sean sostenibles. Al respecto, se observa la importancia de revisar los parámetros que hacen visible el carácter de reciprocidad social a fin de distinguir las cualidades de confort, de seguridad y de sanidad.

Responder a ciertas pautas que hace que los proyectos de inversión revelen la pertinencia o no de las iniciativas institucionales tiene que ver con la definición de criterios en la esfera de la re-configuración de las ciudades. La caracterización de los relacionamientos que se suscitan en los suburbios es una opción para medir la mejora de las condiciones de inclusión y adaptación urbana.

El esquema que se presenta en este trabajo se sustenta bajo la coherencia de los preceptos de habitabilidad, de competitividad y de nuevo urbanismo que sugieren una dimensión disciplinaria para estudiar el desempeño social y productivo de las ciudades de zonas metropolitanas.

\section{Fundamento teórico}

\section{Habitabilidad: un principio a favor de la sustentabilidad}

La habitabilidad hace referencia a la dimensión del hábitat como el lugar donde habitan

\footnotetext{
${ }^{1}$ Desde la economía y la ecología, el término sostenible se refiere a algo "que se puede mantener durante largo tiempo sin agotar los recursos o causar grave daño al medio ambiente" (RAE, 2018: s/p).

${ }^{2}$ La LGCA mencionada pertenece al Cuerpo Académico «Gestión y Evaluación de Objetos de Diseño» que se encuentra registrada ante la Secretaría de Educación Pública desde diciembre del 2015, actualmente con el estatus En Consolidación.
} 
los individuos con alguna satisfacción en lo cultural y lo territorial, y al habitar que refiere a costumbres sociales (Gordillo, 2015 en Alvarado, Adame y Sánchez, 2017). Landázuri y Mercado (2004, en: Moreno, 2008) definen el concepto de habitabilidad desde dos perspectivas: la habitabilidad interna o habitabilidad en el interior de la vivienda y la habitabilidad externa que se refiere al siguiente nivel sistémico o entorno urbano inmediato, en donde se involucra la accesibilidad a servicios y equipamientos. Por su parte, Alcalá (2007) lo refiere al espacio urbano como una condición habitacional donde la vivienda está integrada físicamente a la ciudad, con buena accesibilidad a servicios y equipamientos, rodeada de un espacio público de calidad (Moreno, 2008).

Diversos autores se han propuesto estudiar la categoría de 'cohabitar' en zonas de uso público en ciudades metropolitanas desde los principios de la sustentabilidad. Esto como aquella condición en que se comparten espacios públicos habitables. La ONU-Habitat (2016, p. 4) señala, a través de los objetivos de Desarrollo Sostenible \& la Iniciativa de Ciudades Prósperas3 en el numeral 11 "conseguir que las ciudades y los asentamientos humanos sean inclusivos, seguros, resilientes y sostenibles", en cuyos indicadores se refiere a proporcionar acceso a transporte seguros, a redoblar esfuerzos para proteger y salvaguardar el patrimonio cultural y natural del mundo, a proporcionar acceso universal a zonas verdes y espacios públicos seguros, inclusivos y accesibles, y a apoyar los vínculos económicos, sociales y ambientales positivos entre las zonas urbanas, periurbanas y rurales fortaleciendo la planificación del desarrollo nacional y regional (ONU-Habitat, 2016, p. 4).

Desde la perspectiva del Estado mexicano se busca que las ciudades y los asentamientos humanos sean competitivos. Dicho escenario se manifiesta en la Agenda 2030 del Gobierno del Estado de México y en el Programa Sectorial de Desarrollo Social 2013-2018, vinculado con la meta nacional intitulada México Incluyente.4

Los ejes fundamentales del hábitat buscan intensificar el bienestar cultural. Es indudable la dificultad que existe para aplicar estándares que permitan dimensionar en qué medida se cumple el derecho que tienen todos los ciudadanos de participar en la propiedad del territorio urbano dentro de parámetros democráticos, de justicia social y de condiciones ambientales sustentables, tal como se declara en la Carta Mundial del Derecho a la Ciudad5 (Foro Social de la Américas, Foro Mundial Urbano, Foro Social Mundial, 2012).

\footnotetext{
3 http://unhabitat.org/downloads/es/mexico/cpil603/ODS_CPI.pdf

4 Objetivo 2: "Construir un entorno digno que propicie el desarrollo a través de la mejora de los servicios básicos, la calidad y espacios de vivienda y la infraestructura social." Objetivo 4: Construir una sociedad igualitaria donde exista acceso irrestricto al bienestar social mediante acciones que protejan el ejercicio de los derechos de todas las personas." Objetivo 5: "Fortalecer la participación social para impulsar el desarrollo comunitario a través de esquemas de inclusión productiva y cohesión social” (SEDESOL, s/a: p. 43).

${ }^{5}$ Documento emitido por la ONU-Hábitat en el Foro Social de las Américas - Quito, 2004; Foro Mundial Urbano - Barcelona, 2004; y Foro Social Mundial - Porto Alegre, 2005. El instrumento se fundamenta en tres ejes: El ejercicio de los derechos humanos que aseguran el bienestar colectivo de los habitantes y la producción y gestión social del hábitat; la gestión democrática de la ciudad, a través de la participación de la sociedad de forma directa y participativa, en el planeamiento y gobierno de las ciudades, fortaleciendo las administraciones públicas a escala local, así como las organizaciones sociales; y la función social de la propiedad y de la ciudad, siendo predominante el bien común sobre el derecho individual de propiedad, lo que implica el uso socialmente justo y ambientalmente sustentable del espacio urbano (Mathivet, 2009).
} 
La habitabilidad es un principio que se ha construido a partir de políticas, regulaciones y normas institucionales que proveen marcos legales efectivos a favor de la calidad de vida urbana, cuya condición se interpreta analizando el sentido de la calidad ambiental, el sentido de bienestar y el sentido de la identidad cultural. Particularmente, la habitabilidad en las ciudades metropolitanas del valle de Toluca ha manifestado del año 1970 al 2015 un crecimiento exponencial con la inclusión de 22 municipios (GEM, s/a).

Reconocer las directrices que el diseño -en cualquiera de sus manifestaciones - debe atender a favor de la configuración natural o artificial de los espacios comunes es relevante para monitorear y reportar lo necesario en función de la conexión funcional y simbólica del individuo con su medio. Pérez (1999, en: Moreno, 2008: p.51) lo refiere como las "condiciones óptimas que se conjugan y que determinan sensaciones de confort en lo biológico y psicosocial dentro del espacio donde el hombre habita y actúa".

Bently, Alcock, Murrain, McGlynn y Smith (2008) exponen, en su estudio responsive environments, que los entornos exitosos y aceptados por los usuarios tienen que ver con los principios de permeabilidad, variedad, legibilidad, robustez y riqueza. Junto con estos también hacen referencia tanto el grado en el que los usuarios ponen su propio sello en un determinado lugar [personalización] como la apariencia del espacio que hace que la gente sea consciente de las características que presenta el sitio [apropiación].

La interconexión de esos principios deriva en otros factores, por ejemplo en la calidad de la habitabilidad urbana cuando los individuos concretan la relación con sus espacios a partir del reconocimiento del universo simbólico, funcional y estético. Segovia (2007) expone que es a través de la intensidad de las relaciones sociales cuando se facilita la capacidad de acoger y mezclar distintos grupos y comportamientos. Lynch (1960) añade que los lugares dejan de ser vistos sólo como entidades funcionales cuando pasan a ser elementos para formar identidades. De acuerdo con Pérez, dichas identidades están íntimamente vinculadas al grado de satisfacción de los servicios y a la percepción del espacio habitable como sano, seguro y grato visualmente (Pérez, 1999 en: Moreno, 2008).

\section{Competitividad urbana: señales a favor del crecimiento inclusivo}

La palabra competir se origina de la expresión latina cum petere que significa «buscar juntos» comprometiendo al otro que también existe e interviene (RAE, 2018). A la par de esta expresión, la competitividad se circunscribe entre las exigencias impuestas por el desarrollo tecnológico y la capacidad global. Lo que hace entonces posible mantener escenarios sostenibles es el crecimiento inclusivo y el valor de los recursos materiales tanto tangibles como aquellos intangibles. Estos últimos se identifican como capital humano [know how, capacidades y experiencia técnicas], capital relacional [conexiones, fidelidad y participación con el exterior] y capital estructural [sistemas, políticas, valores culturales, investigación y desarrollo].

La competitividad urbana en ciudades semi diversificadas y diversificadas se enfoca a partir de argumentos económicos y estratégicos que son subordinados a las condiciones que la evolución demográfica origina (Cabrero, Orihuela y Zincardi, 2009). Particularmente la entidad mexiquense, en el 2015 albergaba más de 16 millones de habitantes con una densidad promedio 
de 724 habitantes por kilómetro cuadrado; de esa población 73.3\% presenta al menos una carencia social (INEGI, 2014) en donde es evidente, de acuerdo con Quiróz, Salgado y Miranda (2012: p. 8), "una alta concentración de población y de actividades económicas", a diferencia de la "zona sur-sureste donde la carencia de infraestructura y servicios limita su desarrollo".

Los asuntos de tipo económico se identifican con "factores de producción, infraestructura, localización y amenidades" y los argumentos estratégicos se observan mediante "elementos ligados a la política pública, estrategia urbana, cooperación entre los sectores público y privado y diseño institucional." (Cabrero, Orihuela y Zincardi, 2009: s/p). Ambos casos afectan al crecimiento inclusivo y provocan todo tipo de inconvenientes socio cultural en el desempeño de la estructura tradicional de barrios, toda vez que la identidad de las ciudades representa un símbolo de resguardo y de asociación de intereses nacionales e individuales.

En la relación social del hábitat es pertinente entender aquellas expresiones que la diversidad de sus colectivos urbanos provoca en los escenarios donde lo circundante debe tener un absoluto sentido de pertenencia. Esto tiene injerencia en las escalas de confort y prosperidad, de salud ambiental y de seguridad ciudadana que al reservarse a otros ejercicios se limita las respuestas socio-culturales resilientes. Es importante entonces que se reconozcan los efectos y significaciones que los espacios comunes suscitan en los usuarios bajo algunos preceptos como la libertad, la equidad, la solidaridad, la dignidad y la justicia social.

\section{Nuevo urbanismo: apuntes a favor del espacio común}

Tal como lo define Schteingart (1989) existen una serie de mecanismos y actores sociales que intervienen constantemente en el medio edificado y van modificando su comportamiento y sus formas de producción que afectan el entorno urbano. Se entiende que la configuración de las ciudades, como el lugar donde el hombre establece las relaciones que dan sentido a los patrones en el espacio y la espacialidad que genera su hábitat (De Hoyos, 2010) es el resultado de la suma de conexiones que la población realiza en un territorio.

El Nuevo Urbanismo se originó en la década de los 90 como una manera de planear las ciudades desde el punto de vista sustentable en lo referente a aspectos urbanos (Hernández, 2008). Es un concepto que refiere al estudio de la peatonización en las ciudades, a la conectividad urbana, a la diversidad del uso del suelo, a la diversidad en materia de vivienda, a la calidad en arquitectura y diseño urbano, a la estructura tradicional de barrios y colonias, y al incremento en la densidad urbana. Factores que están en estrecha relación con la competitividad urbana y con fenómenos sociales vinculados con el hábitat.

Desde el 2008, la Comisión para la Cooperación Ambiental lo reconoce como una arquitectura que en sus prácticas utiliza procesos y materiales que minimizan la huella de carbono demandando soluciones que impactan desde la planeación y la intervención del sitio integrando el diseño, la construcción, la operación y el mantenimiento -hasta la demolición de las infraestructuras. Abonce (2007) hace hincapié en el concepto de crecimiento inteligente y en las redes de participación urbana cuyos preceptos bien pueden obedecer a los fundamentos del diseño urbano y arquitectónico desde el enfoque sustentable. 
Por otra parte, destaca la importancia de favorecer la calidad en la arquitectura y el diseño urbano apreciando las interacciones en situaciones de seguridad, de salubridad y de confort (Moreno, 2008) en el contexto de suburbios inclusivos y mejor adaptados en urbes metropolitanas. Dicha condición hace mirar hacia una infraestructura que favorezca la movilidad y la conectividad con un sentido de percepción espacial y mayor grado de confianza colectiva. Entendemos entonces que la identidad de las ciudades representa un símbolo de resguardo y de asociación de intereses; por ello es pertinente distinguir aquellas expresiones que los colectivos urbanos provocan en ciertos escenarios donde lo circundante suscita un absoluto sentido incluyente.

Cabe mencionar que hay otros elementos que también participan en la sana interacción de dichos sitios, por ejemplo las pautas de la bioconstrucción que presenta las características siguientes: (s/a, 2011, p: 1)

- "Ubicación adecuada: correcta orientación solar; evitar campos electromagnéticos; evitar campos eléctricos; estudio geobiológico del asentamiento; evitar alteraciones geológicas, fallas y corrientes de agua.

- Materiales: ciclo de vida sostenible / extracción respetuosa; reducida transformación; cercanos al lugar; naturales; reciclables; reciclados y biocompatibles.

- Eficiencia energética: captación solar; inercia térmica; aislamiento adecuado; integración energías renovables; sistema de aclimatación natural.

- Gestión de residuos y gestión eficiente de agua.

- Estructura y cerramientos: bloques y ladrillos de tierra cocida; bloques de tierra estabilizada; tierra prensada y adobes; madera y piedra.

- Paramentos y morteros: cal hidráulica y cal grasa; yeso, arcilla y madera.

- Aislantes: fibras naturales; arcilla expandida.

- Acabados: pinturas de silicato, de arcilla y a la cal.

- Conducción de aguas: polietileno de alta densidad; polibutileno.

- Bajantes: polietileno, polipropileno; cerámica, hierro fundido; acero galvanizado.

- Desagües: cobre, barro cocido; polipropileno.

- Pavimentos: barro cocido; suelos continuos de mortero, madera; bambú; linóleo.”

Los principios emitidos en la Carta del Nuevo Urbanismo con representación de una amplia base de ciudadanos [compuesta por líderes del sector público y privado, activistas comunitarios y profesionales multidisciplinarios] están comprometidos en restablecer la relación entre el arte de construir y el de hacer comunidad, a través de la planificación y el diseño con base en la participación ciudadana. Entre sus postulados indica que "la revitalización de lugares urbanos depende de cuán seguros sean. El diseño de vías y edificios deberían reforzar entornos seguros, pero no a expensas de la accesibilidad y apertura"; "las calles y plazas deben ser seguras, cómodas e interesantes para el peatón. Correctamente configuradas fomentan el caminar y permiten a los vecinos conocerse y proteger sus comunidades" (Congreso para el Nuevo urbanismo, s/p). ${ }^{6}$

\footnotetext{
${ }^{6}$ Traducción al español de documento original Charter of the New Urbanism, aprobado por El Congreso para el Nuevo Urbanismo. Recuperado en: https://www.google.com.mx/search?ei=C6InXab7CJjVtAbbmJOQCQ\&q=carta+del+nuevo+urbanismo\&oq=carta+ del+nuevo+urbanismo\&gs_l=psyab.3..0j0i22i30.296257.308516..308844...0.0..0.177.2341.24j3 .....4....1..gwswiz......0i71j0i131j0i67j0i67i70i249.wXEzWOeGaQA
} 
En el caso del valle de Toluca, y derivado de la industrialización del corredor TolucaLerma, en la década de los 60s inició un proceso de metropolización y no fue hasta dos décadas después que se consideró como metrópoli semidiversificada. (GEM, s/a). La región está compuesta por 15 municipios; las ciudades que integran esta zona metropolitana son: Almoloya de Juárez, Almoloya del Río, Atizapán, Calimaya, Capulhuac, Chapultepec, Lerma, Metepec, Mexicaltzingo, Ocoyoacac, Otzolotepec, Rayón, San Antonio la Isla, San Mateo Atenco, Temoaya, Tenango del Valle, Texcalyacac, Tianguistenco, Toluca, Xalatlaco, Xonacatlán y Zinacantepec.

\section{Método}

Desde un enfoque de orden cualitativo se abordó el trabajo bajo el método de teoría fundamentada, el cual permite, mediante ciertos procedimientos interpretativos y de codificación construir inductivamente un conocimiento sobre un fenómeno. En un primer momento se recopiló y analizó la información de conceptos, a partir de los cuales se formaron los constructos que dieron lugar a la definición de categorías y a su interpretación.

A fin de comprender el derecho de cohabitar espacios comunes en condiciones sostenibles, se estudiaron las teorías de la habitabilidad, la competitividad urbana y el nuevo urbanismo. Estos conceptos formaron una referencia teórica desde donde analizar el desempeño y la significación de las ciudades de zonas metropolitanas. A partir de esto, se definieron las categorías y su interpretación, lo cual funge de insumo para el diseño de indicadores que son necesarios para evaluar el desempeño del entorno público urbano.

Las categorías y dimensiones se estructuraron siguiendo las aportaciones de Bently, et.at. (2008) bajo los ejes de permeabilidad, de variedad, de robustez, de legibilidad y de riqueza [Véase la figura 1]. De estos ejes se desprendieron las categorías con las cuales se podrá medir e interpretar el desempeño y los significados de los espacios públicos que se producen desde la experiencia en ciudades de zonas metropolitanas del valle de Toluca.

Se advierten ciertos fenómenos que establecen las condiciones que son regulares y que sirven para explicar el comportamiento de las escalas territoriales. Esto según la capacidad de satisfacer la integridad y la equidad de los individuos en su cohabitar cotidiano en sitios ausentes de amenazas de orden fisiológico y antropológico.

A partir de reconocer conceptualmente las variables del estudio, se definieron los referentes teóricos que son compatibles en el marco de desarrollo sustentable para exponer el desempeño de la habitabilidad y la competitividad urbana:

- Dimensión social: formación, comunidad, implicación, inclusión, información, participación ciudadana, redistribución, equidad, cohesión social, uso del espacio público, identidad y pertenencia;

- Dimensión económica: generación de ingresos, ahorro de costes en la acción pública, subordinación al interés general midiendo la eficacia en relación a los objetivos y la eficiencia en relación con los procesos; y 
- Dimensión ambiental: consumo de recursos y gestión ambiental.

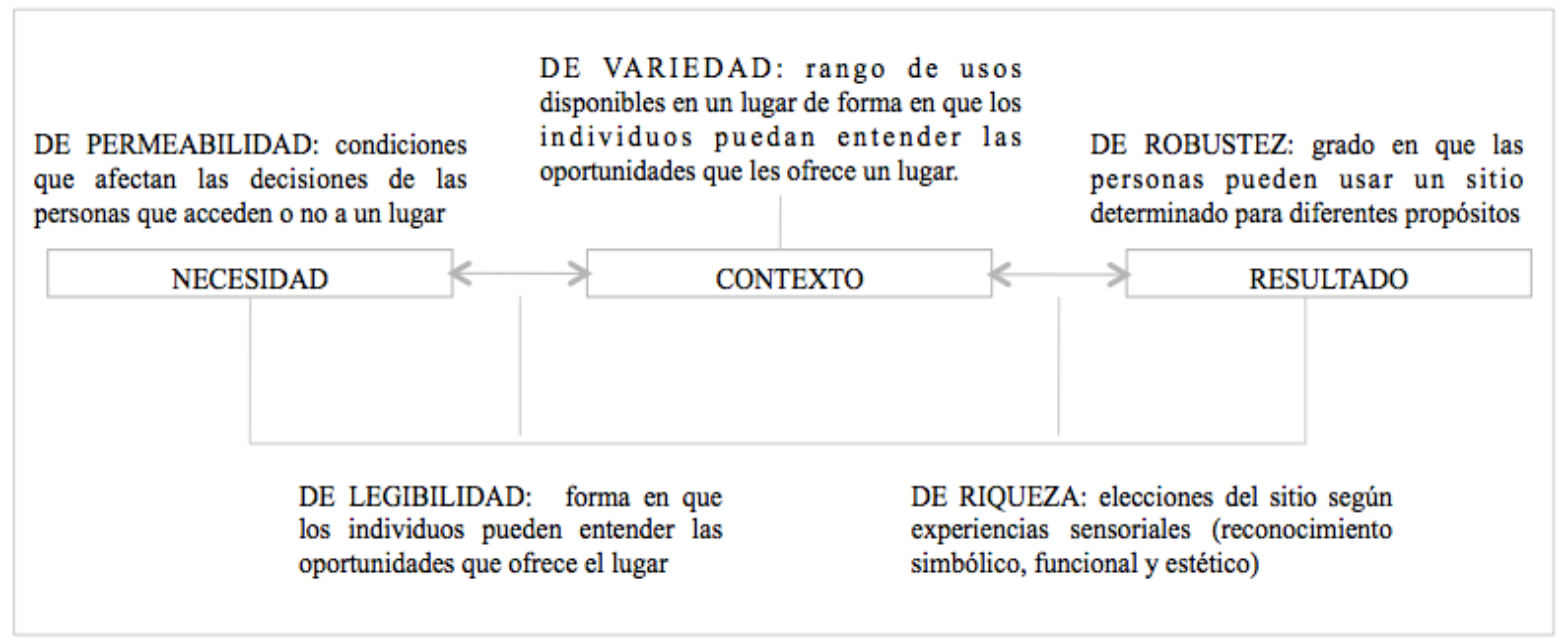

Figura 1. Descripción de los ejes para el estudio de la habitabilidad en el entorno urbano Fuente: Elaboración propia con base en Bently, et.al., 2008.

\section{Resultados}

\section{Categorías para medir e interpretar el desempeño de espacios comunes en ciudades de zonas metropolitanas}

\section{a. Eje de Permeabilidad}

La calidad de la permeabilidad, tal como lo exponen Bently, et al. (2008) responde a las rutas alternativas que ofrece un lugar para conectarse con otro a fin de crear un entorno receptivo, así como a la visibilidad de las mismas. Adicionalmente, vale entenderla como "la relación que existe entre la introducción visual y la densidad del campo espacial que se puede atravesar o filtrar corporalmente, visualmente, olfativamente, acústicamente.’7

Bajo esta perspectiva se busca hacer una comparación de las necesidades satisfechas y los comportamientos que se presentan entre grupos sociales distintos en una misma condición de movilidad. El código para reconocer el derecho de los individuos en el marco de la equidad social es la imparcialidad ciudadana que se entiende como la "aplicación de los derechos y obligaciones de manera justa y equitativa a las personas, independientemente de la clase social o género al que pertenezcan." 8 Asimismo, es propicio que se registren aquellos problemas y otras restricciones de interacción que se presentan en los circuitos del entorno público.

7 https://ehecatlteoria.wordpress.com/2011/10/05/conceptos-milian/

8 https://www.significados.com/equidad/ Consultado en octubre de 2018 
Tabla 1.

Categorías de medición del eje de permeabilidad

\section{Dimensión sustentable}

Social: capacidad de acoger y movilizar todo tipo de grupos de acuerdo con los comportamientos y las necesidades específicas de la población usuaria.

Económica: competencia para formar comunidades socio-productivas con independencia funcional para mejorar los esquemas de consumo en los centros urbanos.

Ambiental: capacidad de modificar las condiciones que provocan daños al ambiente y de acciones para mitigarlas.

\section{Interpretación}

Características del diseño de espacios inclusivos y menos jerárquicos.

Tipo de funciones y actividades de la población usuaria.

Nivel de movilización de la población usuaria.

Incremento en la escala de desarrollo y de la apropiación satisfactoria de espacios públicos y privados.

$\square \quad$ Satisfacción de usuarios para desarrollar actividades productivas en el lugar.

Grado de segregación vehicular y de peatones.

Nivel de limpieza y mantenimiento de la infraestructura del sitio.

Fuente: elaboración propia.

\section{b. Eje de Variedad}

La variedad urbana se refleja en la diversidad de experiencias y usos que ofrece un lugar. Se entiende como la "cualidad intrínseca de la ciudad relacionada directamente con la calidad de vida e imprescindible para plantear modelos alternativos de intervención en la ciudad y el territorio, así como procesos de regeneración y rehabilitación integral” (González, 2013).

Esta condición hace referencia a una "política que busque integrar a todas las personas en la sociedad para que éstas puedan participar y contribuir en ella y beneficiarse en este proceso". 9

En esta categoría se busca medir el rango de usos disponibles que provee el lugar de acuerdo con la demanda de oportunidades que requieren los individuos. Su alcance ha de admitir que los efectos de la eficiencia de las políticas públicas, de la capacidad de los servicios y de la significación de la infraestructura física y material se consigan precisar. Intervienen otros factores en el estudio de la variedad para medir su desempeño en función del impacto social inclusivo atendiendo, por ejemplo, circunstancias de equidad de género y de equidad de capacidades físicas, discriminación por indigencia y segregación étnica o religiosa bajo la perspectiva de cohesión social.

9 https://www.significados.com/inclusion// Consultado en octubre de 2018. 
Tabla 2. Categorías de medición del eje de variedad

\begin{tabular}{lll}
\hline \multicolumn{1}{c}{ Dimensión sustentable } & \multicolumn{1}{c}{ Interpretación } \\
\hline $\begin{array}{l}\text { Social: capacidad de inclusión } \\
\text { ciudadana que persigue una causa justa } \\
\text { de habitabilidad con fines de interés } \\
\text { compartido como símbolo de resguardo } \\
\text { y de asociación de intereses. }\end{array}$ & $\square \quad \begin{array}{l}\text { Potencial en cuanto a la incidencia de niveles de } \\
\text { demanda para diferentes usos. } \\
\text { Nivel de seguridad de habitabilidad. }\end{array}$ \\
$\begin{array}{l}\text { Económica: competitividad circundante, } \\
\text { diferenciación y afectación de espacios } \\
\text { comerciales financieramente factibles. }\end{array}$ & $\square$ & $\begin{array}{l}\text { Diversificación de espacios asequibles y de fácil } \\
\text { acceso. } \\
\text { Combinación de usos comerciales posibles } \\
\text { (hipermercado, supermercado, almacén, pequeños }\end{array}$ \\
puntos de venta). & $\begin{array}{l}\text { Grado de apropiación de políticas y programas de } \\
\text { tipo sustentable. }\end{array}$ \\
$\begin{array}{l}\text { Ambiental: capacidad de mantener } \\
\text { actividades en función de ello. }\end{array}$ & $\square$
\end{tabular}

Fuente: elaboración propia

\section{c. Eje de Robustez}

Esta expresión se refiere a una condición de tipo sostenible que es representada mediante la construcción y la adecuación de estructuras y espacios cuya cualidad sea la diversidad de su uso en diferentes tiempos y espacios; impacta incluso el apoyo de la tecnología para su adaptación a nuevas funciones (De Schiller y Evans, 2006 en Alvarado, et. al, 2017).

Mediante esta categoría se trata de conocer el grado en que las personas pueden hacer uso de un sitio determinado con diferentes intenciones. Esto a través de la medición del cambio esperado en una situación y población objetivo determinada una vez que la acción se lleva a cabo. Interviene el ciclo de vida que "define las etapas que pasa un producto desde su nacimiento hasta su declive". ${ }^{10}$ El ciclo de vida y su relación con el usuario se entiende como el paquete de políticas y prácticas para incorporar a la sociedad productos, procesos y servicios nuevos o mejorados, cuya aplicación se refleja en el aprovechamiento de los recursos materiales y naturales.

Tabla 3. Categorías de medición del eje de robustez

\begin{tabular}{ll}
\multicolumn{1}{c}{ Dimensión sustentable } & \multicolumn{1}{c}{ Interpretación } \\
\hline $\begin{array}{l}\text { Social: capacidad de guardar orden y } \\
\text { justicia social en términos de protección, } \\
\text { asilo, auxilio, amparo y defensa. }\end{array}$ & $\square \begin{array}{l}\text { Grado de satisfacción ciudadana en temas de seguridad } \\
\text { pública. } \\
\text { Grado de satisfacción ciudadana en medidas de asistencia } \\
\text { y resguardo. }\end{array}$ \\
$\begin{array}{l}\text { Económica: pertenencia en términos de } \\
\text { estabilidad y garantía de patrimonio } \\
\text { comercial vecinal, comunitario o regional. }\end{array}$ & $\begin{array}{l}\text { Ciclo de vida de espacios con funciones diversificadas } \\
\text { protegiendo las estructuras tradicionales de barrios y } \\
\text { ciudades. }\end{array}$
\end{tabular}

\footnotetext{
${ }^{10}$ https://www.significados.com/ciclo de vida// Consultado en octubre de 2018.
} 


\section{Dimensión sustentable} de bienes y servicios de manera responsable para minimizar el impacto ambiental.

\section{Interpretación}

Fuente: elaboración propia

\section{d. Eje de Legibilidad}

En el campo del diseño, la legibilidad se refiere a la facilidad con que las personas pueden entender un lugar de acuerdo con las cualidades que son comprensibles en los niveles de forma física y patrones de actividad (Bently, et. al., 1984).

Desde el campo de la gestión es pertinente acercarse al conjunto de oportunidades que puedan contrastar la política a un cierto objetivo implícito en la concreción de un programa de desarrollo social o urbano. La legibilidad se aplica, desde la acción pública, en la subordinación al interés general midiendo la eficacia en relación con los objetivos y la eficiencia en relación con los procesos.

Tabla 4.

Categorías de medición del eje de legibilidad

\section{Dimensión sustentable}

Social: capacidad de gestión pública y privada de recursos sociales en cuanto al enfoque espacial de sitios dignos.

Económica: capacidad de gestión pública y privada de recursos productivos.

Ambiental: capacidad de gestión pública y privada de imagen urbana diseñada bajo las premisas de la sustentabilidad.
Balance en la eficiencia en los sistemas de limpieza en calles y sitios públicos.

Balance en la eficiencia en sistemas de acopio de residuos sólidos en espacios públicos y privados.
Fuente: elaboración propia

\section{e. Eje de Riqueza}

Desde el campo del diseño, el eje de riqueza se identifica con la variedad de experiencias sensoriales, principalmente aquellas que sentimos cuando vemos y comprendemos el entorno. Sin embargo, desde el enfoque de la gestión, la participación ciudadana -reconocida como "un derecho legítimo de todo ciudadano para intervenir en la toma de decisiones, impulsando así el 
desarrollo local y la democracia participativa"11 -expresa poder detectar tantas situaciones problemáticas existan a fin de exponer posibles oportunidades de solución. Esto en el marco de los compromisos que los individuos adoptan para contribuir favorablemente ante su propia realidad para proteger sus derechos. Su significación se adhiere a una causa justa que como usuarios de las ciudades se manifiesta de manera solidaria.

Tabla 5.

Categorías de medición del eje de riqueza

\begin{tabular}{lll}
\hline \multicolumn{1}{c}{ Dimensión sustentable } & \multicolumn{1}{c}{ Interpretación } \\
\hline $\begin{array}{l}\text { Social: capacidad de promover programas } \\
\text { ciudadanos bajo las características de } \\
\text { responsabilidad social, empatía y } \\
\text { confianza. }\end{array}$ & $\square \quad \begin{array}{l}\text { Nivel de participación de la sociedad civil. } \\
\text { Tipo de acciones públicas de la ciudadanía, de empresas y } \\
\text { de entidades de gobierno. }\end{array}$ \\
$\begin{array}{l}\text { Económica: capacidad de distribución } \\
\text { equitativa y justa de los bienes } \\
\text { patrimoniales de la ciudad que resulta de } \\
\text { las actividades productivas que se } \\
\text { desarrollan. }\end{array}$ & $\square \quad \begin{array}{l}\text { Distribución de los bienes patrimoniales públicos entre los } \\
\text { diferentes estratos sociales. }\end{array}$ \\
$\begin{array}{l}\text { Ambiental: capacidad de enfrentar } \\
\text { resultados que deriven en la tendencia de } \\
\text { proyectos de inversión sostenible. }\end{array}$ & $\square \quad \begin{array}{l}\text { Gapacidad de la población para identificar los impactos } \\
\text { negativos que causa el deterioro ambiental. }\end{array}$ \\
\hline
\end{tabular}

\section{Conclusiones}

La población civil y las instituciones públicas enfrentan una serie de situaciones problemáticas que, a raíz de la expansión de las ciudades del valle de Toluca, se han manifestado en la calidad de las interrelaciones urbanas, en la compleja participación y apropiación del espacio y en la falta de identidad cultural del sitio, entre otras. A fin de entender su efecto, se juzgó conveniente precisar aquellas variables que suponen el derecho de cohabitar espacios comunes en condiciones sostenibles.

Dicho argumento se alinea con nuevos paradigmas que impulsa la medición que hace visible el nivel de desempeño y de significación de los lugares, al tiempo de aportar información útil para beneficiar la toma de decisiones en torno a la viabilidad, comprensión, eficiencia y eficacia de los proyectos urbanos.

Con base en los conceptos de habitabilidad, de competitividad y de nuevo urbanismo, se buscó la significación del espacio público en función de los ejes de permeabilidad, de variedad, de robustez, de legibilidad y de riqueza que aportaron las pautas para observar a las ciudades con base en su posición frente a los pilares de la sustentabilidad. Es entonces que las categorías de medición presentadas inducen a la recuperación del ejercicio primordial que el espacio público debe aportar a la población en el contexto de ciudades mejor adaptadas a las demandas locales y regionales. Se pueden designar ya sea desde la estructura urbana de manzana y calle hasta la dimensión de pueblos, barrios y colonias.

\footnotetext{
${ }^{11}$ https://www.significados.com/participacionciudadana// Consultado en octubre de 2018.
} 
Para lograr el funcionamiento integral en las ciudades, se deduce que las áreas de uso comunitario deben incorporar dichos factores a fin de beneficiar el derecho social de convivencia y de habitabilidad que los individuos requieren para desenvolverse de manera óptima en los espacios públicos que proveen las metrópolis.

Desde la dimensión sustentable se definieron 15 categorías que se interpretaron en 30 clases. Esto quiere decir que se presenta un escenario que es propicio para abordar con mayor precisión la cualificación del estatus social, económico y ambiental en el ejercicio de los espacios públicos de las ciudades. Se desdobla la conveniencia de insistir en un discurso, iniciado por la onu-Habitat, a razón de provocar una mayor consideración por parte de los colectivos civiles por modificar y adaptar ciertos patrones de movilidad, de gestión pública y privada, de diversidad e inclusión, de identidad, de competitividad y de seguridad en las interrelaciones de los espacios públicos. Asimismo, se extiende la perspectiva hacia la diversidad del uso del suelo y al privilegio de zonas verdes y de recreación. Todas éstas en el entendido de fortalecer la concentración de la actividad cívica, institucional y comercial.

La observación de cada uno de los escenarios en donde lo circundante cobra sentido, aporta información que influye para identificar el rango en el nivel de correspondencia, en tanto a la calidad que resulta del efecto que un sitio público provoca entre sus colectivos respecto a su seguridad y a su progreso.

El estudio en su totalidad no termina con esta precisión; prosigue la construcción de un sistema de indicadores de carácter cuantitativo y cualitativo sobre los espacios públicos de ciudades de zonas metropolitanas que pueda ser común para los municipios del valle de Toluca, y cuya aplicación permita comparar una serie de diagnósticos que se efectúen con cierto nivel de afinidad. Esto se enfrenta como una estrategia para lograr que las decisiones públicas tengan un fundamento crítico y mejor argumentado para que ciudades de zonas metropolitanas logren ser sostenibles y resilientes en el menor plazo posible.

\section{Bibliografía}

Alvarado, A., Adame, M., Sánchez, N. (2017). Habitabilidad urbana en el espacio público, el caso del centro histórico de Toluca, Estado de México. Sociedad y Ambiente, núm. 13, pp. 129169. ISSN: 2007-6576. Recuperado de: http://redalyc.org/articulo.oa?id=455752309007

Avonce, M. (2007). El nuevo urbanismo: un desafío para las ciudades metropolitanas del siglo XXI. Cuadernos de Arquitectura y Nuevo Urbanismo, núm. 02, año 1. Instituto Tecnológico de Estudios Superiores de Monterrey.

Bently, I., Alcock, A., Murrain, P., McGlynn, S., Smith, G. (2008). Responsive Environments. A manual for Designers. Oxford: Elsevier, ltd.

Cabrero, M., Orihuela, J., Zincardi, C. (2009). Competitividad urbana en México: una propuesta de medición. Revista Eure, Vol. XXXV, núm. 106, pp. 79-99. ISSN 0250-7161. Recuperado de: http://dx.doi.org/10.4067/S0250-71612009000300005

De Hoyos, M. (2010). La casa: Origen de la conformación territorial. Aportaciones epistemológicas al estudio del territorio. Toluca, México: Secretaría de Educación del 
Gobierno del Estado de México. ISBN: 978-607-495-082-3

S/A (2011). Qué es la bioconstrucción. Pautas y materiales. Valencia: Ecohabitar. Recuperado de: http://www.ecohabitar.org/bioconstruccion-pautas-y-materiales/.

Foro Social de las Américas, Foro Mundial urbano, Foro Social Mundial. (2012). Carta Mundial por el derecho a la Ciudad. Revista paz y conflictos, núm. 5, ISBN: 1988-7221.

GEM. Gobierno del Estado de México (s/a). Sistema Estatal de Información Urbana, Metropolitana y Vivienda. Estado de México. Recuperado de: http://plataforma.seduym.edomex.gob.mx/SIGZonasMetropolitanas/PEIM/antecede.do 9 de julio de 2019.

González, G. (2013). Una aproximación a la definición de variedad urbana desde la complejidad: aplicación al análisis urbanístico de tres barrios madrileños. Tesis de doctorado. Universidad Politécnica de Madrid, Centro de lectura: ETS Arquitectura, Departamento de Urbanística y Ordenación del Territorio. / Centro de realización: ETS Arquitecto. Madrid, España.

Hernández, M. (2008). Introducción al urbanismo sustentable o nuevo urbanismo. Espacios Públicos, vol. 11, núm. 23, pp. 298-307. Toluca: Universidad Autónoma del Estado de México. ISSN: 1665-8140. Recuperado de: http://www.redalyc.org/articulo.oa?id=67611217015

INEGI. Instituto Nacional de Geografía y Estadística (2014). Indicadores por entidad federativa. México: INEGI. Recuperado de: https://www.inegi.org.mx/app/estatal/?ag=15; consultado en enero de 2019.

Lynch, K. (1960). La imagen de la ciudad. Barcelona: Gustavo Gili, SL. Traducción al castellano, 1998. España.

Mathivet, Ch. (2009). Historia del derecho a la ciudad: una propuesta que va más allá de un nuevo concepto. Dosier derecho a la ciudad. Recuperado de: http://base.d-ph.info/es/fiches/dph/fiche-dph-8034.html.

Moreno, O. S. H. (2008). La habitabilidad urbana como condición de vida. Palapa, vol. III, núm. II, pp. 47-54; Universidad de Colima, México. http://redalyc.uaemex.mx/src/inicio/ArtPdfRed.jsp?iCve=94814774007

ONU-Hábitat. (2016). Informe sobre el Índice de Prosperidad Urbana. La iniciativa de la prosperidad urbana. Programa de Naciones Unidas para los Asentamientos Humanos por un mejor fututo urbano. Recuperado de: https://es.unhabitat.org/cpi-mexico/

Quiroz, C., Salgado, V., Miranda, G. (2012). Crecimiento urbano y diversificación económica en el Estado de México, 1990-2007. Revista Análisis Económico, vol. XXVII Recuperado de:<http://vifww.redalyc.org/articulo.oa?id=41324594002> ISSN 0185-3937

RAE. Real Academia Española. (2018) Diccionario de la lengua española. Recuperado de: https://dle.rae.es/?id=DgIqVCc.

Schteingart, M. (1989). Los productores del espacio habitable. Estado, empresa y sociedad en la Ciudad de México. México: El Colegio de México.

SEDESOL. Secretaría de Desarrollo Social del Gobierno de México. (S/a). Programa Sectorial de Desarrollo Social 2013-2018. Gobierno de la República Mexicana. Recuperado de: www.sedesol.gob.mx

Segovia, O. (2007). Espacios públicos urbanos y construcción social: una relación de correspondencia. Espacios públicos y construcción social. Hacia un ejercicio de ciudadanía. Santiago de Chile: Ediciones SUR. 